Vietnam Journal of Mechanics, VAST, Vol. 31, No. 3 \&4 (2009), pp. 233-246

\title{
CONSTRUCTION AND ANALYSIS OF LOCALIZED RESPONSES FOR GRADIENT DAMAGE MODELS IN A 1D SETTING
}

\author{
K. Pham and J.-J. Marigo \\ Université Paris 6, Institut Jean le Rond d'Alembert, \\ 4 Place Jussieu 75005 Paris
}

\begin{abstract}
We propose a method of construction of non homogeneous solutions to the problem of traction of a bar made of an elastic-damaging material whose softening behavior is regularized by a gradient damage model. We show that, for sufficiently long bars, localization arises on sets whose length is proportional to the material internal length and with a profile which is also characteristic of the material. We point out the very sensitivity of the responses to the parameters of the damage law. All these theoretical considerations are illustrated by numerical examples.
\end{abstract}

\section{INTRODUCTION}

It is possible to give an account of rupture of materials with damage models by the means of the localization of the damage on zones of small thickness where the strains are large and the stresses small. However the choice of the type of damage model is essential to obtain valuable results. Thus, local models of damage are suited for hardening behavior but cease to give meaningful responses for softening behavior. Indeed, in this latter case the boundary-value problem is mathematically ill-posed (Benallal et al. [1], Lasry and Belytschko, [5]) in the sense that it admits an infinite number of linearly independent solutions. In particular damage can concentrate on arbitrarily small zones and thus failure arises in the material without dissipation energy. Furthermore, numerical simulation with local models via Finite Element Method are strongly mesh sensitive. Two main regularization techniques exist to avoid these pathological localizations, namely the integral (Pijaudier-Cabot and Bažant [10]) or the gradient (Triantafyllidis and Aifantis [11]) damage approaches, see also [6] for an overview. Both consist in introducing non local terms in the model and hence a characteristic length. We will use gradient models and derive the damage evolution problem from a variational approach based on an energetic formulation. The energetic formulations, first introduced by Nguyen [9] and then justified by Marigo [4] by thermodynamical arguments for a large class of rate independent behavior, constitute a very promising way to treat in a unified framework the questions of bifurcation and stability of solutions to quasi-static evolution problems. Francfort and Marigo [4] and Bourdin, Francfort and Marigo [4] have extended this approach to Damage and Fracture Mechanics. 
Considering the one-dimensional problem of a bar under traction with a particular gradient damage model, Benallal and Marigo [2]apply the variational formulation and emphasize the scale effects in the bifurcation and stability analysis: the instability of the homogeneous response and the localization of damage strongly depend on the ratio between the size of the body and the internal length of the material. The goal of the present paper is to extend a part of the results (the questions of stability will no be investigated) of [2] for a large class of elastic-softening material. Specifically, we propose a general method to construct localized solutions of the damage evolution problem and we study the influence of the constitutive parameters on the response. Several scenarii depending on the bar length and on the material parameters enlighten the size effects induced by the non local term. The paper is structured as follows. Section 2 is devoted to the statement of the damage evolution problem. In Section 3 we describe, perform and illustrate the method of construction of localized solutions and conclude by the different scenarii of responses.

The following notation are used: the prime denotes either the spatial derivative or the derivative with respect to the damage parameter, the dot the time derivative, e.g. $u^{\prime}=\partial u / \partial x, E^{\prime}(\alpha)=d E(\alpha) / d \alpha, \dot{\alpha}=\partial \alpha / \partial t$.

\section{SETTING OF THE DAMAGE PROBLEM}

\subsection{The gradient damage model}

We consider a one-dimensional gradient damage model in which the damage variable $\alpha$ is a real number growing from 0 to $1, \alpha=0$ is the undamaged state and $\alpha=1$ is the full damaged state. The behavior of the material is characterized by the state function $W_{\ell}$ which gives the energy density at each point $x$. It depends on the local strain $u^{\prime}(x)$ ( $u$ denotes the displacement and the prime stands for the spatial derivative), the local damage value $\alpha(x)$ and the local gradient $\alpha^{\prime}(x)$ of the damage field at $x$. Specifically, we assume that $W_{\ell}$ takes the following form

$$
W_{\ell}\left(u^{\prime}, \alpha, \alpha^{\prime}\right)=\frac{1}{2} E(\alpha) u^{\prime 2}+w(\alpha)+\frac{1}{2} E_{0} \ell^{2} \alpha^{\prime 2}
$$

where $E_{0}$ represents the Young modulus of the undamaged material, $E(\alpha)$ the Young modulus of the material in the damage state $\alpha$ and $w(\alpha)$ can be interpreted as the density of the energy dissipated by the material during a homogeneous damage process (i.e. a process such that $\alpha^{\prime}(x)=0$ ) where the damage variable of the material point grows from 0 to $\alpha$. The last term in the right hand side of (1) is the "non local" part of the energy which plays, as we will see later, a regularizing role by limiting the possibilities of localization of the damage field. For obvious reasons of physical dimension, it involves a material characteristic length $\ell$ that will fix the size of the damage localization zone. The local model associated with the gradient model consists in setting $\ell=0$ and hence in replacing $W_{\ell}$ by $W_{0}$ :

$$
W_{0}\left(u^{\prime}, \alpha\right):=\frac{1}{2} E(\alpha) u^{\prime 2}+w(\alpha) .
$$

The qualitative properties of the (gradient or local) model, in particular its softening or hardening character, strongly depend on some properties of the stiffness function $\alpha \mapsto$ $E(\alpha)$, the dissipation function $\alpha \mapsto w(\alpha)$, the compliance function $\alpha \mapsto S(\alpha)=1 / E(\alpha)$ 
and their derivatives. From now on we will adopt the following hypothesis, the importance of which will appear later:

Hypothesis 1 (Constitutive assumptions). $\alpha \mapsto E(\alpha)$ and $\alpha \mapsto w(\alpha)$ are non negative and continuously differentiable with $E(1)=0, w(0)=0, E^{\prime}(\alpha)<0$ and $w^{\prime}(\alpha)>0$ for all $\alpha \in[0,1)$. Moreover $-w^{\prime}(\alpha) / E^{\prime}(\alpha)$ is increasing to $+\infty$ while $w^{\prime}(\alpha) / S^{\prime}(\alpha)$ is decreasing to 0 when a grows from 0 to 1 .

Example 1. A particularly interesting family of models which satisfy the assumptions above is the following one

$$
E(\alpha)=E_{0} \frac{(1-\alpha)^{q}}{(1+\alpha)^{p}}, \quad w(\alpha)=(p+q) \frac{\sigma_{0}^{2}}{2 E_{0}} \alpha
$$

where $p \geq 1$ and $q \geq 1$ are two constants playing the role of constitutive parameters and $\sigma_{0}$ represents the critical stress of the material.

\subsection{The damage problem of a bar under traction}

Let us consider a homogeneous bar whose natural reference configuration is the interval $(0, L)$ and whose cross-sectional area is $S$. The bar is made of the nonlocal damaging material characterized by the state function $W_{\ell}$ given by (1). The end $x=0$ of the bar is fixed, while the displacement of the end $x=L$ is prescribed to a non negative value $U_{t}$

$$
u_{t}(0)=0, \quad u_{t}(L)=U_{t} \geq 0, \quad t \geq 0
$$

where, in this quasi-static setting, $t$ denotes the loading parameter or shortly the "time", $u_{t}$ is the displacement field of the bar at time $t$. The evolution of the displacement and of the damage in the bar is obtained via a variational formulation, the main ingredients of which are recalled hereafter, see [2] for details.

Let $\mathcal{C}_{U_{t}}$ and $\mathcal{D}$ be respectively the kinematically admissible displacement fields at time $t$ and the convex cone of admissible damage fields:

$\mathcal{C}_{U_{t}}=\left\{v: v(0)=0, v(L)=U_{t}\right\}, \quad \mathcal{C}_{0}=\{v: v(0)=0, v(L)=0\}, \quad \mathcal{D}=\{\beta: \beta(x) \geq 0, \forall x\}$

where $\mathcal{C}_{0}$ is the linear space associated with $\mathcal{C}_{U_{t}}$. The precise regularity of these fields is not specified here, we will simply assume that there are at least continuous and differentiable everywhere. Then with any admissible pair $(u, \alpha)$ at time $t$, we associate the total energy of the bar

$$
\begin{aligned}
\mathcal{P}(u, \alpha) & :=\int_{0}^{L} W_{\ell}\left(u^{\prime}(x), \alpha(x), \alpha^{\prime}(x)\right) S d x \\
& =\int_{0}^{L}\left(\frac{1}{2} E(\alpha(x)) S u^{\prime}(x)^{2}+w(\alpha(x)) S+\frac{1}{2} E_{0} S \ell^{2} \alpha^{\prime}(x)^{2}\right) d x
\end{aligned}
$$

For a given initial damage field $\alpha^{0}$, the damage evolution problem reads as:

For each $t>0$, find $\left(u_{t}, \alpha_{t}\right)$ in $\mathcal{C}_{U_{t}} \times \mathcal{D}$ such that

For all $(v, \beta) \in \mathcal{C}_{\dot{U}(t)} \times \mathcal{D}, \quad \mathcal{P}^{\prime}\left(u_{t}, \alpha_{t}\right)\left(v-\dot{u}_{t}, \beta-\dot{\alpha}_{t}\right) \geq 0$ 
with the initial condition $\alpha_{0}(x)=\alpha^{0}(x)$. In $(7), \mathcal{P}^{\prime}(u, \alpha)(v, \beta)$ denotes the derivative of $\mathcal{P}$ at $(u, \alpha)$ in the direction $(v, \beta)$ and is given by

$$
\mathcal{P}^{\prime}(u, \alpha)(v, \beta)=\int_{0}^{L}\left(E(\alpha) S u^{\prime} v^{\prime}+\left(\frac{1}{2} E^{\prime}(\alpha) S u^{\prime 2}+w^{\prime}(\alpha) S\right) \beta+E_{0} S \ell^{2} \alpha^{\prime} \beta^{\prime}\right) d x
$$

The set of admissible displacement rates $\dot{u}$ can be identified with $\mathcal{C}_{\dot{U}(t)}$, while the set of admissible damage rates $\dot{\alpha}$ can be identified with $\mathcal{D}$ because the damage can only increase for irreversibility reasons. Inserting in (7) $\beta=\dot{\alpha}_{t}$ and $v=\dot{u}_{t}+w$ with $w \in \mathcal{C}_{0}$, we obtain the variational formulation of the equilibrium of the bar,

$$
\int_{0}^{L} E\left(\alpha_{t}(x)\right) u_{t}^{\prime}(x) w^{\prime}(x) d x=0, \quad \forall w \in \mathcal{C}_{0}
$$

From (8), we deduce that the stress along the bar is homogeneous and is only a function of time

$$
\sigma_{t}^{\prime}=0, \quad \sigma_{t}=E\left(\alpha_{t}(x)\right) u_{t}^{\prime}(x), \quad \forall x \in(0, L)
$$

Dividing (9) by $E\left(\alpha_{t}\right)$, integrating over $(0, L)$ and using boundary conditions (4), we find

$$
\sigma_{t} \int_{0}^{L} S\left(\alpha_{t}(x)\right) d x=U_{t}
$$

The damage problem is obtained after inserting (8)-(10) into (7). That leads to the variational inequality governing the evolution of the damage

$$
-\sigma_{t}^{2} \int_{0}^{L} S^{\prime}\left(\alpha_{t}\right) \beta d x+\int_{0}^{L} 2 w^{\prime}\left(\alpha_{t}\right) \beta d x+\int_{0}^{L} 2 E_{0} \ell^{2} \alpha_{t}^{\prime} \beta^{\prime} d x \geq 0
$$

where the inequality must hold for all $\beta \in \mathcal{D}$ and becomes an equality when $\beta=\dot{\alpha}_{t}$. After an integration by parts and using classical tools of the calculus of variations, we find the strong formulation for the damage evolution problem: For (almost) all $t \geq 0$,

Irreversibility condition:

$$
\dot{\alpha}_{t} \geq 0
$$

Damage criterion:

$$
-\sigma_{t}^{2} S^{\prime}\left(\alpha_{t}\right)+2 w^{\prime}\left(\alpha_{t}\right)-2 E_{0} \ell^{2} \alpha_{t}^{\prime \prime} \geq 0
$$

Loading/unloading condition:

$$
\dot{\alpha}_{t}\left(-\sigma_{t}^{2} S^{\prime}\left(\alpha_{t}\right)+2 w^{\prime}\left(\alpha_{t}\right)-2 E_{0} \ell^{2} \alpha_{t}^{\prime \prime}\right)=0 .
$$

Remark 1. We can deduce also from the variational approach natural boundary conditions and regularity properties for the damage field. In particular, we obtain that $\alpha_{t}^{\prime}$ must be continuous everywhere. As boundary conditions at $x=0$ and $x=L$ we will simply take $\alpha_{t}^{\prime}(0)=\alpha_{t}^{\prime}(L)=0$ although the more general ones induced by the variational principle correspond to a combination of inequalities and equalities like (13)-(14). These regularity properties of the damage field (and consequently the boundary conditions) hold only for the gradient model $(\ell \neq 0)$ and disappear for the local model $(\ell=0)$. As long as the regularity in time is concerned, we will only consider evolution such that $t \mapsto \alpha_{t}$ is at least continuous. 


\subsection{The homogeneous solution and the issue of uniqueness}

If we assume that the bar is undamaged at $t=0$, i.e. if $\alpha^{0}(x)=0$ for all $x$, then it is easy to check that the damage evolution problem admits the so-called homogeneous solution where $\alpha_{t}$ depends on $t$ but not on $x$. Let us construct this particular solution in the case where the prescribed displacement is monotonically increasing, i.e. when $U_{t}=t L$. From (10), we get $\sigma_{t}=E\left(\alpha_{t}\right) t$. Inserting this relation into (13) and (14) leads to

$$
\frac{t^{2}}{2} \leq-\frac{w^{\prime}\left(\alpha_{t}\right)}{E^{\prime}\left(\alpha_{t}\right)}, \quad \dot{\alpha}_{t}\left(\frac{t^{2}}{2}+\frac{w^{\prime}\left(\alpha_{t}\right)}{E^{\prime}\left(\alpha_{t}\right)}\right)=0 .
$$

Since $\alpha_{0}=0, \alpha_{t}$ remains equal to 0 as long as $t \leq \varepsilon_{0}=\sqrt{-2 w^{\prime}(0) / E^{\prime}(0)}$. That corresponds to the elastic phase. For $t>\varepsilon_{0}$, since $-w^{\prime} / E^{\prime}$ is increasing by virtue of Hypothesis 1 , the first relation of (15) must be an equality. Therefore $\alpha_{t}$ is given by

$$
\alpha_{t}=\left(-\frac{w^{\prime}}{E^{\prime}}\right)^{-1}\left(\frac{t^{2}}{2}\right)
$$

and grows from 0 to 1 when $t$ grows from $\varepsilon_{0}$ to $\infty$. During this damaging phase, the stress $\sigma_{t}$ is given by

$$
\sigma_{t}=\sqrt{\frac{2 w^{\prime}\left(\alpha_{t}\right)}{S^{\prime}\left(\alpha_{t}\right)}}
$$

Since $w^{\prime} / S^{\prime}$ is decreasing to 0 by virtue of Hypothesis $1, \sigma_{t}$ decreases to 0 when $t$ grows from $\varepsilon_{0}$ to $\infty$. This last property corresponds to the softening character of the damage model. Note that $\sigma_{t}$ tends only asymptotically to 0 , what means that an infinite displacement is necessary to break the bar in the case of a homogeneous response.

The non local term has no influence on the homogeneous solution which is solution both for the gradient and the local damage models. Let us now examine the issue of the uniqueness of the response. In the case of the local damage model, it is well known that the evolution problem admits an infinite number of solution. Does the gradient term force the uniqueness? The answer to this fundamental question essentially depends on the ratio $\ell / L$ of the internal length with the bar length, as it is proved in [?] in the case $p=2$, $q=0$. Specifically it was shown that the homogeneous solution is the unique solution of the evolution problem when $\sigma_{0} L \leq \pi E_{0} \ell$, i.e. when the bar is small enough, while there exists an infinite number of solutions otherwise. However, when the bar is long enough, although the number of solutions is infinite, the fundamental difference between the local and the gradient models is that the length of the damaged zone is bounded from below for the gradient model while it can be chosen arbitrarily small for the local model. The main goal of the next section is to extend these results for a large class of gradient models and to study the properties of non homogeneous solutions.

Let us remark that any solution of the evolution problem contains the same elastic phase, i.e. $\alpha_{t}=0$ as long as $t \leq \varepsilon_{0}$. Therefore, localizations can appear only when $t>\varepsilon_{0}$. 


\section{NON HOMOGENEOUS SOLUTIONS OF THE DAMAGE PROBLEM}

\subsection{The method of construction of non homogeneous solutions}

Let us consider one solution of the evolution problem. Setting

$$
\sigma_{0}:=\sqrt{\frac{2 w^{\prime}(0)}{S^{\prime}(0)}}=E(0) \varepsilon_{0}
$$

we deduce from (13), that $0 \leq \sigma_{t} \leq \sigma_{0}$. Indeed, $\sigma_{t} \geq 0$ by virtue of (4) and (10). Then, integrating (13) over $(0, L)$ and using the boundary conditions $\alpha_{t}^{\prime}(0)=\alpha_{t}^{\prime}(L)=0$, we obtain

$$
\sigma_{t}^{2} \int_{0}^{L} S^{\prime}\left(\alpha_{t}(x)\right) d x \leq \int_{0}^{L} 2 w^{\prime}\left(\alpha_{t}(x)\right) d x .
$$

But, since $w^{\prime} / S^{\prime}$ is a decreasing function of $\alpha$ by virtue of Hypothesis 1 and since $\alpha_{t} \geq 0$ by virtue of the irreversibility condition, we have

$$
2 w^{\prime}\left(\alpha_{t}(x)\right) \leq \sigma_{0}^{2} S^{\prime}\left(\alpha_{t}(x)\right), \quad \forall x \in(0, L) .
$$

Integrating over $(0, L)$ and inserting the result into $(17)$ gives $\sigma_{t}^{2} \leq \sigma_{0}^{2}$. Therefore $\sigma_{0}$ is the maximal stress that the material can sustain.

The point of departure in the construction of localized damage solutions is to seek for solutions for which the equality in (13) holds only in some parts of the bar. For a given $t>\varepsilon_{0}$, the localized damage field will be characterized by its set $\mathcal{S}_{t}=\bigcup_{i} \mathcal{S}_{t}^{i}$ of localization zones $\mathcal{S}_{t}^{i}$ where $\mathcal{S}_{t}^{i}$ is an open interval of $[0, L]$ of the form $\left(x_{i}-D_{t}, x_{i}+D_{t}\right)$ (the independence of its length on $i$ will be proved). In $[0, L] \backslash \mathcal{S}_{t}$, the material is supposed to be sound and therefore these parts will correspond to elastic zones where $\alpha_{t}=0$. By sake of simplicity, we will not consider localization zones centered at the boundary of $(0, L)$, i.e. with $x_{i}=0$ or $x_{i}=L$. Therefore, we force the damage to vanish at $x=0$ and $x=L$. The successive steps of the construction are as follows:

(1) For a given $t$, assuming that $\sigma_{t}$ is known, we determine the profile of the damage field in a localization zone;

(2) For a given $t$, we obtain the relation between $\sigma_{t}$ and $U_{t}$;

(3) We check the irreversibility condition.

\subsection{Damage profile in a localization zone}

Since $t$ is fixed, we omit the index $t$ in all quantities which are time-dependent. Let $\sigma \in\left(0, \sigma_{0}\right)$ be the supposed known stress and $\mathcal{S}_{i}=\left(x_{i}-D, x_{i}+D\right)$ be a putative localization zone. The damage field $\alpha$ must satisfy

$$
-\sigma^{2} S^{\prime}(\alpha)+2 w^{\prime}(\alpha)-2 E_{0} \ell^{2} \alpha^{\prime \prime}=0 \quad \text { in } \mathcal{S}_{i} .
$$

Since we assume by construction that the localization zone is matched to an elastic zone and since $\alpha$ and $\alpha^{\prime}$ must be continuous, see Remark 1, the damage field has also to satisfy the boundary conditions

$$
\alpha\left(x_{i} \pm D\right)=\alpha^{\prime}\left(x_{i} \pm D\right)=0 .
$$

Multiplying (18) by $\alpha^{\prime}$ and integrating with respect to $x$, we obtain the first integral

$$
-\sigma^{2} S(\alpha)+2 w(\alpha)-E_{0} \ell^{2} \alpha^{2}=C \quad \text { in } \mathcal{S}_{i}
$$


where $C$ is a constant. Using (19) and Hypothesis 1 , we get $C=-\sigma^{2} / E_{0}$ and (20) can read as

$$
\ell^{2} \alpha^{\prime}(x)^{2}=H(\sigma, \alpha(x)) \text { in } \quad \mathcal{S}_{i}
$$

with

$$
E_{0} H(\sigma, \beta):=2 w(\beta)-\sigma^{2}\left(S(\beta)-\frac{1}{E_{0}}\right) \quad \text { for } \quad \beta \in[0,1) .
$$

Since $E_{0} \frac{\partial H}{\partial \beta}(\sigma, \beta)=2 w^{\prime}(\beta)-\sigma^{2} S^{\prime}(\beta)$ and since, by virtue of Hypothesis $1, w^{\prime}(\beta)>0$ and $1-\frac{\sigma^{2} S^{\prime}(\beta)}{2 w^{\prime}(\beta)}$ decreases from $1-\sigma^{2} / \sigma_{0}^{2}>0$ to $-\infty$ when $\beta$ grows from 0 to $1, H$ is first increasing from 0 , then decreasing to $-\infty$. Hence, there exists a unique positive value of $\beta$, say $\bar{\alpha}(\sigma)$, where $H$ vanishes:

$$
H(\sigma, \bar{\alpha}(\sigma))=0, \quad 0<\bar{\alpha}(\sigma)<1 .
$$

$\bar{\alpha}(\sigma)$ corresponds to the maximal value of the damage (at the given time), taken at the center of the localization zone. It depends only on $\sigma$ and enjoys the following property:

Property 1 (The dependence on the stress of the maximal value of the damage in the localization zone). When $\sigma$ decreases from $\sigma_{0}$ to $0, \bar{\alpha}(\sigma)$ increases from 0 to 1 .

Proof. Indeed, let $0<\sigma_{1}<\sigma_{2} \leq \sigma_{0}$. Since $0=H\left(\sigma_{1}, \bar{\alpha}\left(\sigma_{1}\right)\right)=H\left(\sigma_{2}, \bar{\alpha}\left(\sigma_{2}\right)\right)<H\left(\sigma_{1}, \bar{\alpha}\left(\sigma_{2}\right)\right)$, and, since $H\left(\sigma_{1}, \beta\right)<0$ when $\bar{\alpha}\left(\sigma_{1}\right)<\beta<1$, we have $\bar{\alpha}\left(\sigma_{1}\right)>\bar{\alpha}\left(\sigma_{2}\right)$. Hence $\sigma \mapsto \bar{\alpha}(\sigma)$ is decreasing. Since $\partial H / \partial \beta\left(\sigma_{0}, \beta\right)<0$ for $\beta>0$, we have $\bar{\alpha}\left(\sigma_{0}\right)=0$. Let us prove that $\lim _{\sigma \rightarrow 0} \bar{\alpha}(\sigma)=1$. Let $\alpha_{m}=\lim _{\sigma \rightarrow 0} \bar{\alpha}(\sigma)$ (the limit exists and is positive since $\bar{\alpha}(\sigma)$ is decreasing). If $\alpha_{m}<1$, passing to the limit in (23) when $\sigma$ goes to 0 gives $0=H\left(0, \alpha_{m}\right)=2 w\left(\alpha_{m}\right)$, a contradiction. Hence $\alpha_{m}=1$.

The size of the localization zone is deduced from (21) by integration. It depends also on $\sigma$ and is given by

$$
D(\sigma)=\ell \int_{0}^{\bar{\alpha}(\sigma)} \frac{d \beta}{\sqrt{H(\sigma, \beta)}} .
$$

$D(\sigma)$ is proportional to the internal length and is finite because the integral is convergent. (Indeed, $H(\sigma, \beta)$ behaves like $\frac{\partial H}{\partial \beta}(\sigma, 0) \beta$ near $\beta=0$ and like $\frac{\partial H}{\partial \beta}(\sigma, \bar{\alpha}(\sigma))(\beta-\bar{\alpha}(\sigma))$ near $\beta=\bar{\alpha}(\sigma)$. Since $\frac{\partial H}{\partial \beta}(\sigma, 0)>0$ and $\frac{\partial H}{\partial \beta}(\sigma, \bar{\alpha}(\sigma))<0$, the integral is convergent.) Provided that $L \geq 2 D(\sigma)$, it is really possible to insert a localization zone of size $2 D(\sigma)$ inside the bar. Concerning the dependence of $D(\sigma)$ on $\sigma$, we obtain the following fundamental property the proof of which is not given here (it is based on a careful study of the behavior of the integral giving $D(\sigma))$ :

Property 2 (Dependence on the stress of the size of the localization zone.). The size $D(\sigma)$ of the localization zone varies continuously with $\sigma, D\left(\sigma_{0}\right)$ and $D_{0}=\lim _{\sigma \downarrow 0} D(\sigma)$ are finite and given by

$$
D\left(\sigma_{0}\right)=\pi \ell \sqrt{\frac{2 E_{0}}{\sigma_{0}^{2} S^{\prime \prime}(0)-2 w^{\prime \prime}(0)}}, \quad D_{0}=\ell \int_{0}^{1} \sqrt{\frac{E_{0}}{2 w(\alpha)}} d \alpha .
$$


The size function $\sigma \mapsto D(\sigma)$ is not necessarily decreasing. In particular $\frac{d D}{d \sigma}\left(\sigma_{0}\right)<0$ if and only if the following inequality holds

$$
S^{\prime \prime}(0)\left(S^{\prime \prime}(0) \sigma_{0}^{2}-2 w^{\prime \prime}(0)\right)>S^{\prime}(0)\left(S^{\prime \prime \prime}(0) \sigma_{0}^{2}-2 w^{\prime \prime \prime}(0)\right) .
$$

The position $x_{i}$ of the center can be chosen arbitrarily in the interval $[D(\sigma), L-$ $D(\sigma)$ ]. We finally deduce from (21) that, in the localization zone, the damage field is given by the following implicit relation between $x$ and $\alpha$ :

$$
\left|x-x_{i}\right|=\ell \int_{\alpha}^{\bar{\alpha}(\sigma)} \frac{d \beta}{\sqrt{H(\sigma, \beta)}} .
$$

It is easy to see that the damage field is symmetric with respect to the center of the localization zone, decreasing continuously from $\bar{\alpha}(\sigma)$ at the center to 0 at the boundary.

Remark 2. The size of the localization zone and the profile of the damage field inside depends only on $\sigma$. Since $\sigma$ is a global quantity, all the localization zones have the same size and the same profile at a given time. The maximal number of localization zones that can exist at a given time depends on the length of the bar: the longer the bar, the greater the maximal number of localization zones.

Example 2. In the case of the family of models introduced in Example 1 with $q \geq 1$ and $p \geq 1$ the size of the localization zone at $\sigma=\sigma_{0}$ or 0 are given by

$$
D\left(\sigma_{0}\right)=\frac{\pi \ell \sqrt{2}}{\varepsilon_{0} \sqrt{(p+q)^{2}+q-p}}, \quad D_{0}=\frac{2 \ell}{\varepsilon_{0} \sqrt{p+q}} .
$$

The necessary condition (26) of growing of the localization zone when the stress decreases reads as $(q-p)^{2}>(q+p)^{2}(q-p+2)$. It is in particular satisfied for $q=2$ and $p \geq 4$, but it is never satisfied when $q \geq 1$ and $1 \leq p \leq 2$.

\subsection{The force-displacement relation}

The time is fixed and we still omit the index $t$. Let $U$ be the prescribed displacement, $\varepsilon=U / L$ the average strain and $\sigma$ the stress in the bar which contains $n \geq 1$ localization zones. Using (10), recalling that $\alpha=0$ outside the localization zones and that all localization zones have the same size $2 D(\sigma)$ and the same profile, we get

$$
U=\sigma \int_{0}^{L} S(\alpha(x)) d x=\sigma\left(n \int_{\mathcal{S}_{1}} S(\alpha(x)) d x+\frac{L-2 n D(\sigma)}{E_{0}}\right)
$$

The integral $\int_{\mathcal{S}_{1}} S(\alpha(x)) d x$ can be transformed into an integral over the range of $\alpha$ by using (21). Indeed, by symmetry it can reads as $2 \int_{x_{1}-D(\sigma)}^{x_{1}} S(\alpha(x)) d x$. Making the change of variables $x \rightarrow \alpha$, since $\ell d \alpha=\sqrt{H(\sigma, \alpha)} d x$, we obtain

$$
\int_{\mathcal{S}_{1}} S(\alpha(x)) d x=2 \ell \int_{0}^{\bar{\alpha}(\sigma)} \frac{S(\alpha) d \alpha}{\sqrt{H(\sigma, \alpha)}} .
$$

Recalling (24), we finally obtain the overall stress-strain relation

$$
\varepsilon=\varepsilon^{e}(\sigma)+n \frac{\ell}{L} \varepsilon_{1}^{d}(\sigma)
$$




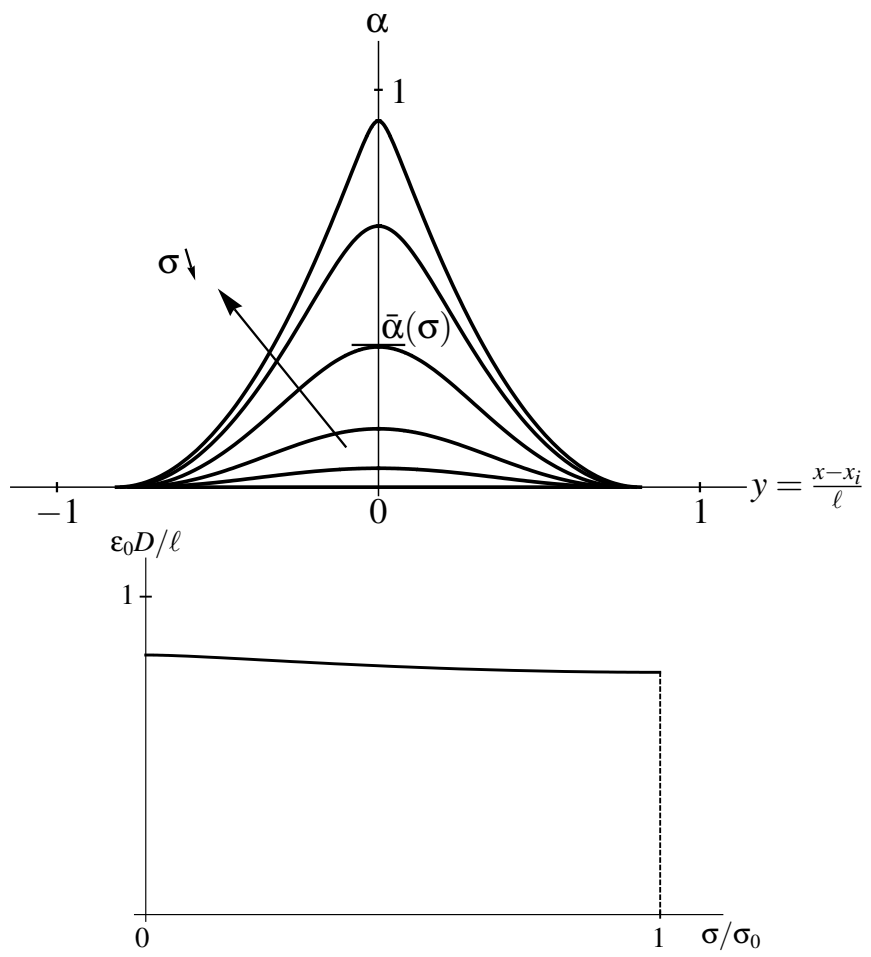

Fig. 1. Left: The damage profile for a given $\sigma$ and its evolution for different $\sigma$ in the case of the model of Example 1 with $q=2$ and $p=4$ (the lower $\sigma$, the higher $\alpha$ ). Right: We check numerically that $\sigma \mapsto D(\sigma)$ is decreasing.

with

$$
\varepsilon^{e}(\sigma)=\frac{\sigma}{E_{0}}, \quad \varepsilon_{1}^{d}(\sigma)=2 \sigma \int_{0}^{\bar{\alpha}(\sigma)}\left(S(\beta)-\frac{1}{E_{0}}\right) \frac{d \beta}{\sqrt{H(\sigma, \beta)}} .
$$

Remark 3. For a given n, (28) gives the average strain in term of the stress. That corresponds to a curve in the $\varepsilon-\sigma$ plane, parametrized by $\sigma$ varying from 0 to $\sigma_{0}$. The curve $n=0$ is the segment corresponding to the elastic phase. Thus $\varepsilon$ can be decomposed into two terms, one associated with the elastic part of bar, the other with the localization zones. Note that $\sigma \mapsto \varepsilon_{1}^{d}(\sigma)$ depends neither on the length of the bar nor on the internal length of the material.

The properties of monotonicity of the function $\sigma \mapsto \varepsilon_{1}^{d}(\sigma)$ play an important role on the presence of snap-backs in the overall response of the bar, see the next subsection. Since $\varepsilon_{1}^{d}\left(\sigma_{0}\right)=0$ and since $\varepsilon_{1}^{d}(\sigma)>0$ for $\sigma<\sigma_{0}, \sigma \mapsto \varepsilon_{1}^{d}(\sigma)$ is necessarily decreasing in the neighborhood of $\sigma_{0}$. We have in particular

Property 3 (Behavior of $\sigma \mapsto \varepsilon_{1}^{d}(\sigma)$ near $\left.\sigma_{0}\right) \cdot \varepsilon_{1}^{d}\left(\sigma_{0}\right)=0$ and

$$
\frac{d \varepsilon_{1}^{d}}{d \sigma}\left(\sigma_{0}\right)=-\frac{\pi 2^{5 / 2} S^{\prime}(0)^{2} \sigma_{0}^{2} E_{0}^{1 / 2}}{\left(S^{\prime \prime}(0) \sigma_{0}^{2}-2 w^{\prime \prime}(0)\right)^{3 / 2}} .
$$


On the other hand, the behavior of $\varepsilon_{1}^{d}(\sigma)$ when $\sigma / \sigma_{0}$ is small is very sensitive to the constitutive parameters as it is shown in the following example and on Figure 2.

Example 3. In the case of the family of models of Example 1, we have

$$
\sigma_{0} \frac{d \varepsilon_{1}^{d}}{d \sigma}\left(\sigma_{0}\right)=-\frac{\pi 2^{5 / 2}(p+q)^{2}}{\left((p+q)^{2}+q-p\right)^{3 / 2}}, \quad \lim _{\sigma \downarrow 0} \varepsilon_{1}^{d}(\sigma)= \begin{cases}0 & \text { if } q<2 \\ \pi 2^{p / 2} & \text { if } q=2 \\ +\infty & \text { if } q>2 .\end{cases}
$$

Consequently, when $q \leq 2$, the overall strain remains finite when the stress goes to 0 , contrary to the homogeneous response where the strain becomes infinite.

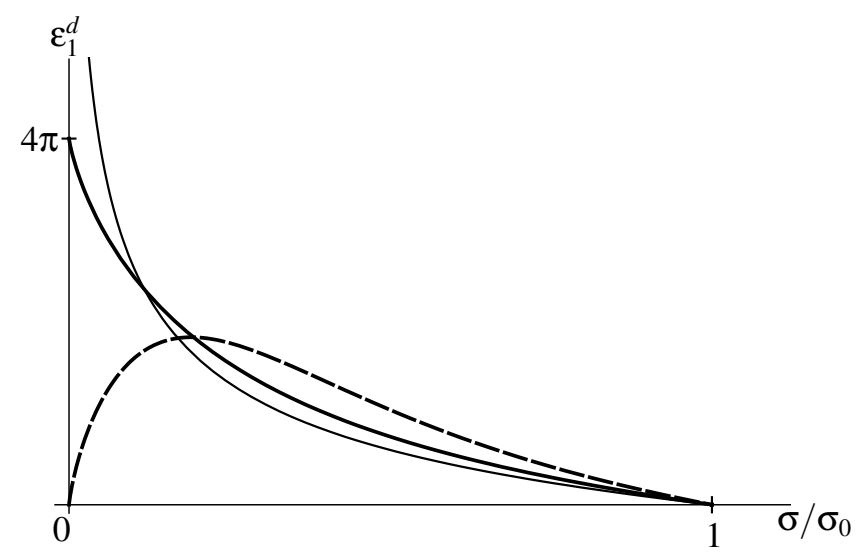

Fig. 2. Graph of the function $\sigma \mapsto \varepsilon_{1}^{d}(\sigma)$ giving the contribution of a localized zone on the overall strain in the case of the model of Example 1 with $p=4$ and different values of $q$ (dashed: $q=1$, thick: $q=2$, thin: $q=3$ ).

\subsection{Checking of the irreversibility}

It remains to check that the localized damage fields that we have constructed at different values of $\sigma$ leads to an evolution in time which satisfies the irreversibility condition $\dot{\alpha} \geq 0$. Let us reintroduce the time and the index $t$ in the notation. Since the center of the localization zone is fixed, the condition of irreversibility is satisfied only if $t \mapsto \bar{\alpha}\left(\sigma_{t}\right)=$ $\alpha_{t}\left(x_{i}\right)$ is not decreasing. Since $\sigma \mapsto \bar{\alpha}(\sigma)$ is decreasing, it is possible only if $t \mapsto \sigma_{t}$ is not increasing. Since $\alpha_{t}\left(x_{i}, x_{i}+D\left(\sigma_{t}\right)\right)=0$ and since $\alpha_{t}(x)>0$ for $\left|x-x_{i}\right|<D\left(\sigma_{t}\right)$ by construction, the condition of irreversibility is satisfied only if $t \mapsto D\left(\sigma_{t}\right)$ is not decreasing. That requires that $\sigma \mapsto D(\sigma)$ is not increasing, condition which is not automatically satisfied by the damage model, see Example 2. When this condition is not satisfied, our construction of the localized solution is no more valid. We must consider an evolution of the damage where a part of the localization zone is unloaded and reenters in a non damaging phase, the size of the still damaging part decreasing with time. To avoid such a situation we make the following hypothesis

Hypothesis 2. We assume that $\alpha \mapsto E(\alpha)$ and $\alpha \mapsto w(\alpha)$ are such that $\sigma \mapsto D(\sigma)$ is decreasing. 
Note that this hypothesis is satisfied in the class of models of Example 1 when $q=2$ and $p \geq 4$. Under this condition, it is possible to obtain the following property

Property 4. Under Hypothesis 2, in order that $t \mapsto \alpha_{t}$ given by (27) in a localization zone (and equal to 0 otherwise) is not decreasing it is necessary and sufficient that $t \mapsto \sigma_{t}$ is not increasing.

Proof. We know that it is necessary, it remains to prove that it is sufficient. Let us assume that $t \mapsto \sigma_{t}$ is not increasing. Then $t \mapsto \bar{\alpha}\left(\sigma_{t}\right)$ and $t \mapsto D\left(\sigma_{t}\right)$ are not decreasing. Let $t_{1}<t_{2}$ and $x$ be such that $\left|x-x_{i}\right| \leq D\left(\sigma_{t_{1}}\right)$. It is sufficient to prove that $\alpha_{2}:=\alpha_{t_{2}}(x) \geq$ $\alpha_{t_{1}}(x)=: \alpha_{1}$. Owing to (27), since $H$ is a decreasing function of $\sigma$ and since $\sigma_{t_{2}} \leq \sigma_{t_{1}}$, we have

$$
0 \leq D\left(\sigma_{t_{2}}\right)-D\left(\sigma_{t_{1}}\right)=\int_{0}^{\alpha_{2}} \frac{\ell d \beta}{\sqrt{H\left(\sigma_{t_{2}}, \beta\right)}}-\int_{0}^{\alpha_{1}} \frac{\ell d \beta}{\sqrt{H\left(\sigma_{t_{1}}, \beta\right)}} \leq \int_{\alpha_{1}}^{\alpha_{2}} \frac{\ell d \beta}{\sqrt{H\left(\sigma_{t_{1}}, \beta\right)}} .
$$

Hence $\alpha_{2} \geq \alpha_{1}$.

By virtue of this last property, our construction of a non homogeneous solution is valid provided that the bar is sufficiently long so that a localization zone can appear and grow without reaching the boundary. Since the size of the localization zone increases with $t$, that leads to the inequality $L \geq 2 n D_{0}$. Owing to (25), that gives the following lower bound for $L$ :

$$
L \geq n \ell \int_{0}^{1} \sqrt{\frac{2 E_{0}}{w(\alpha)}} d \alpha:=n L_{m}>2 n D\left(\sigma_{0}\right) .
$$

Remark 4. Under Hypotheses 1 and 2, we have really obtained a damage evolution $t \mapsto \alpha_{t}$ which satisfies the evolution problem (12)-(14) if the bar is long enough and if we can control the loading in such a manner that the stress is continuously decreasing. But the continuity of $t \mapsto \sigma_{t}$ is not automatically ensured as we show in the next subsection.

\subsection{Size effects and the different scenarii}

Let us consider a loading process where $U_{t}=t L$, i.e. such that the displacement of the end $x=L$ is monotonically increasing. Consequently $t$ corresponds to the average strain of the bar, $\varepsilon=t$, and (28) reads now

$$
t=\frac{\sigma_{t}}{E_{0}}+n \frac{\ell}{L} \varepsilon_{1}^{d}\left(\sigma_{t}\right)
$$

It remains to study under which condition this relation between $t$ and $\sigma$ is invertible. In other words, we have to find when the overall curve $\varepsilon-\sigma$ does not contain snap-backs. Specifically, in order that there is no snap-back, we must have $d \varepsilon / d \sigma(\sigma) \leq 0, \forall \sigma$. By virtue of (28), that gives an upper bound for $L$ :

$$
L \leq n \ell \inf _{\sigma \in\left(0, \sigma_{0}\right]}\left\{-E_{0} \frac{d \varepsilon_{1}^{d}}{d \sigma}(\sigma)\right\}:=n L_{M} .
$$

Of course, this condition is never satisfied when $\sigma \mapsto \varepsilon_{1}^{d}(\sigma)$ is not decreasing. (For example, in the case of the family of models of Example 1, it is never satisfied if $q<2$, cf Example 3.) Depending on the properties of the model, the length of the bar and the number 
of localization zones, we can obtain different scenarii. Let us consider only the situation with one localization zone $(n=1)$, to simplify the presentation. We can distinguish two cases:

(1) Case $L_{m}>2 D\left(\sigma_{0}\right) \geq L_{M}$. In such a case we have three situations

(a) For very short bars, i.e. $L \leq 2 D\left(\sigma_{0}\right)$, no solution with localization is possible. The homogeneous solution is the unique solution;

(b) For short bars, i.e. $2 D\left(\sigma_{0}\right)<L \leq L_{m}$, a solution with localization is possible just after the elastic phase, but the localization zone will progressively cover all the bar and a snap-back is possible;

(c) For long bars, i.e. $L>L_{m}$, a solution with localization is possible, but it is necessarily discontinuous in time because of the presence of a snap-back in the overall stress-strain response.

(2) Case $L_{m}<L_{M}$. We have then four possibilities, the first two (a) and (b) are the same as before

(c) For intermediate bars, i.e. $L_{m}<L \leq L_{M}$, a continuous solution with localization is possible, the damaged zone does not reach the boundary, there is no snap-back;

(d) For long bars, i.e. $L>L_{M}$, a solution with localization is possible, but it is necessarily discontinuous in time because of the presence of a snap-back.

Example 4. In the case of the family of models of Example 1, if $q<2$, then $L_{M}<0$. It is not possible to find a non homogeneous solution without snap-back.

If $q=2$ and $p=4$, then $L_{m}=\frac{4}{\sqrt{6}} \frac{\ell}{\varepsilon_{0}}<\frac{72 \pi}{17 \sqrt{17}} \frac{\ell}{\varepsilon_{0}}=L_{M}$. Therefore, for bars with an intermediate length we can find a continuous in time localized solution, cf Figure 3 (left), while for long bars a localized solution is necessarily discontinuous in time just after the elastic phase, of Figure 3 (right).
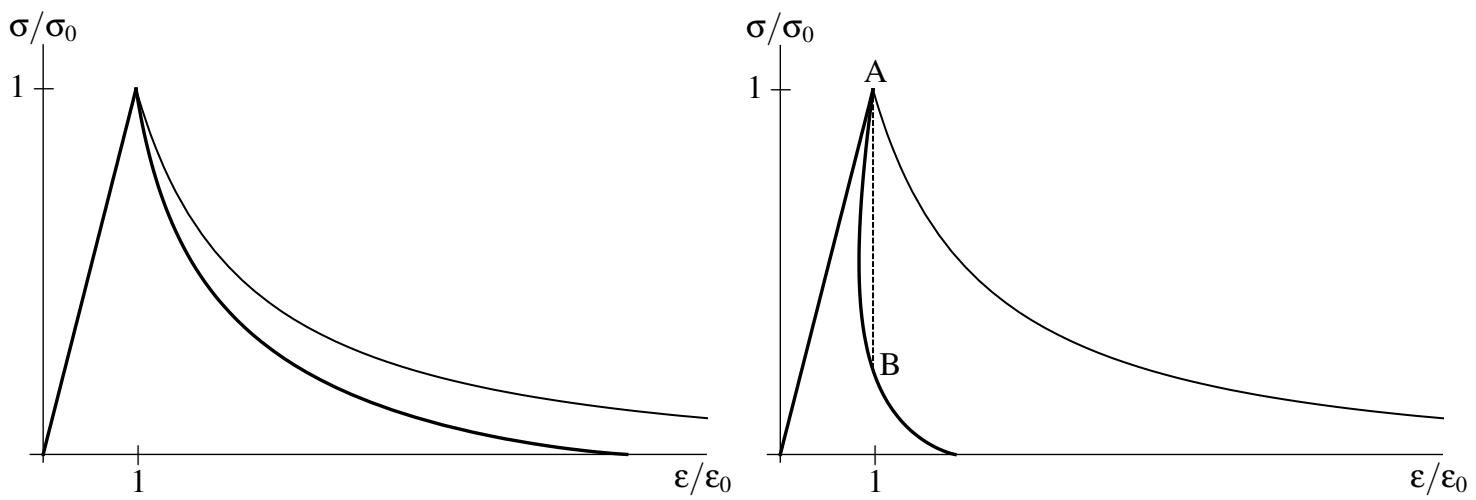

Fig. 3. Overall stress-strain relations for a law of Example 1 with $p=4$ and $q=2$ (Thin curve=homogeneous response; Thick curve=localized response). Left: For a bar of intermediate length; Right: For a long bar. 


\section{CONCLUSION AND PERSPECTIVES}

We have proposed a method of construction of non homogeneous solutions for the one-dimensional damage evolution problem of a bar under traction. We have shown that the properties of such a localized solution is very sensitive to the parameters of the model. This strong dependence could be very interesting from an experimental viewpoint to identify the right law. From a theoretical viewpoint, the presence of the gradient of damage in the model has a regularizing role as expected and limits the possibility of localization of the damage since the size of a localization zone is necessarily greater than a value fixed by the internal length of the material and the others parameters of the model. Moreover, this non local term induces size effects in the response of the bar with in particular a necessarily discontinuous response and even a brutal onset of the damage after the elastic phase for long bars. When the bar is sufficiently long, our construction gives several solutions for the evolution problem, the number of localizations zones being only restricted by the length of the bar. We could probably construct many other solutions as it was made in [?]. This drastic lack of uniqueness that the introduction of a non local term has not removed needs to add a selection criterion. A good candidate is of course the stability criterion introduced in [?] and the next challenge is to find which solutions among all those we have constructed satisfy the stability criterion.

\section{REFERENCES}

[1] A. Benallal, R. Billardon, and G. Geymonat. Bifurcation and localization in rate independent materials. In Q.S. Nguyen, editor, C.S.I.M Lecture Notes on Bifurcation and Stability of Dissipative Systems, Springer-Verlag, 1993.

[2] A. Benallal and J.-J. Marigo. Bifurcation and stability issues in gradient theories with softening, Modelling Simul. Mater. Sci. Eng. 15 (2007) 283295.

[3] B. Bourdin, G.A. Francfort, and J.-J. Marigo, The variational approach to fracture, J. Elasticity, 91:5 148, 2008.

[4] G.A. Francfort and J.-J. Marigo. Stable damage evolution in a brittle continuous medium. Eur. J. Mech. A/Solids 12 (1993) 149 -189.

[5] D. Lasry and Belytschko, Localization limiters in transient problems, Int. J. Solids Structures 24 (1988) 581 - 587.

[6] E. Lorentz and S. Andrieux, Analysis of non-local models through energetic formulations, Int. J. Solids Struct. 40 (2003) 2905 - 2936.

[7] J. -J. Marigo. Constitutive relations in Plasticity, Damage and Fracture Mechanics based on a Work Property, Nuclear Engineering and Design 114 (1989) 249 - 272.

[8] J. -J. Marigo, From Clausius-Duhem and Drucker-Ilyushin inequalities to standard mate- rials. In G. A. Maugin, R. Drouot, and F. Sidoro, editors, Continuum Thermodynamics: the art and science of modelling material behaviour, volume 76 of Solids Mechanics and Its Applications: Paul Germain's anniversary. Kluwer Acad. Publ., 2000.

[9] Q. S. Nguyen, Stability and Nonlinear Solid Mechanics, Wiley \& Son, London, 2000.

$[10]$

[11] G. Pijaudier-Cabot and Z. P. Bazant. Non-local damage theory, J. Eng. Mech. 113 (1987) $1512-1533$.

[12] N. Triantafyllidis and E. C. Aifantis, A gradient approach to localization of deformation: I. hyperelastic materials, J. Elasticity 16 (1986) 225 - 237. 
Received July 13, 2009

\section{XÂY DỰNG VÀ PHÂN TÍCH ĐÁP ƯNG ĐỊA PHƯƠNG HÓA CHO CÁC MÔ HİNH HƯ HỎNG GRADIENT TRONG BÀI TOÁN 1 CHIỀU}

Chúng tôi đề xuất một phương pháp xây dựng lời giải không thuần nhất cho bài toán kéo thanh làm bằng vật liệu đàn hồi bị hư hỏng mà ứng xử mềm hóa được cân đối hóa bằng một mô hình hư hỏng gradient. Chúng tôi chỉ ra rằng, với những thanh đủ dài, sự địa phương hóa tăng lên trên các lớp mà chiều dài của nó tỷ lệ với độ dài vật liệu bên trong và với phân bố cũng là một đặc trưng của vật liệu. Chúng tôi chỉ ra tính rất nhạy cảm của đáp ứng đối với các tham số trong quy luật hư hỏng. Tất cả những nghiên cứu lý thuyết này được minh họa bằng các ví dụ số. 